Int. J. Contemp. Math. Sciences, Vol. 2, 2007, no. 26, 1279 - 1283

\title{
Zero-Divisor Graphs of Idealizations with Respect to Prime Modules
}

\author{
Shahabaddin Ebrahimi Atani \\ Department of Mathematics \\ University of Guilan \\ P. O. Box 1914, Rasht, Iran \\ ebrahimi@guilan.ac.ir, ebrahimiatani@gmail.com \\ Zahra Ebrahimi Sarvandi \\ Department of Mathematics \\ University of Payam Nour, Kerman, Iran
}

\begin{abstract}
Let $R$ be a commutative ring with identity and let $M$ be a prime $R$ module. Let $R(+) M$ be the idealization of the $\operatorname{ring} R$ by the $R$-module $M$. We study the diameter and girth of the zero-divisor graph of the ring $R(+) M$.
\end{abstract}

Mathematics Subject Classification: 13A99, 13A15

Keywords: Zero-divisor, Graph, Prime, Idealization

\section{Introduction}

Throughout All rings are assumed to be commutative rings with non-zero identity. The zero-divisor graph of a ring is the (simple) graph whose vertex set is the set of non-zero zero-divisors, and an edge is drawn between two distinct vertices if their product is zero. This definition is introduced by D. F. Anddeson P. S. Livingston in [1]. In [3] Beck introduced the concept of a zero-divisor graph of a commutative ring. However, he lets all elements of $R$ be vertices of the graph and his work was mostly concerned with coloring of rings. In recent years, the study of zero-divisor graph has grown in various directions. At the heart is the interplay between the ring theoretic properties of a ring and the graph theoretic properties of its zero-divisor graph. The zero-divisor graph of a commutative ring has been studied extensively by several authors, 
e.g. $[1,2,4]$. Our aim in this note is to study the diameter and girth of the zero-divisor graph of the ring $R(+) M$, where $M$ is a prime $R$-module.

Let $R$ be a commutative ring with non-zero identity. We use the notation $A^{*}$ to refer to the non-zero elements of $A$. For two distinct vertices $a$ and $b$ in a graph $\Gamma(R)$, the distance between $a$ and $b$, denoted $\mathrm{d}(a, b)$, is the length of the shortest path connecting $a$ and $b$, if such a path exists; otherwise, $\mathrm{d}(a, b)=\infty$. The diameter of a graph $\Gamma(R)$ is $\operatorname{diam}(\Gamma)=\sup \{d(a, b)$ : $a$ and $b$ are distinct vertices of $\Gamma\}$. We will use use the notation $\operatorname{diam}(\Gamma(R))$ to denote the diameter of the graph of $Z^{*}(R)$. A graph is said to be connected if there exists a path between any two distinct vertices, and a graph is complete if it is connected with diameter one. The girth of a graph $\Gamma$, denoted by $g(\Gamma)$, is the length of a shortest cycle in $\Gamma$, provided $\Gamma$ contains a cycle; otherwise, $g(\Gamma)=\infty$. We will use the notation $g(\Gamma(R))$ to denote the girth of the graph $Z^{*}(R)$.

Let $M$ be an $R$-module. Consider $R(+) M=\{(a, m): a \in R, m \in M\}$ and let $(a, m)$ and $(b, n)$ be two elements of $R(+) M$. Define: $(a, m)+(b, n)=$ $(a+b, m+n)$ and $(a, m)(b, n)=(a b, a m+b n)$. Under this definitions $R(+) M$ becomes a commutative ring with identity. Call this ring the idealization of $M$ in $R$ [5]. Let $M$ be an $R$-module. Then $M$ is called prime if whenever $r m=0$ either $m=0$ or $r M=0$. So $M$ is prime if and only if for every non-zero submodule $N$ of $M$ we have $\left(0:_{R} N\right)=\left(0:_{R} M\right)$. In this case, $P=\left(0:_{R} M\right)$ is a prime ideal of $R$ and we also say that $M$ is a $P$-prime $R$-module.

\section{Diameter and Girth of $\Gamma(R(+) M)$}

Let $M$ be a $P$-prime module over a Commutative ring $R$. The notation below will be kept in this paper: $V_{1}=\left\{(0, m): m \in M^{*}\right\}, V_{2}=\left\{(a, n): a \in P^{*}, n \in\right.$ $M\}$ and $V_{3}=\left\{(a, n): a \in Z^{*}(R), n \in M\right\}$.

Proposition 2.1 Let $R$ be a commutative ring and let $M$ be a P-prime $R$ module. Then:

(i) If $P \neq 0$, then $(a, m) \in Z(R(+) M)$ if and only if $a \in P \cup Z(R)$.

(ii) If $P=0$, then $(a, m) \in Z(R(+) M)$ if and only if $a=0$ and $m \in M^{*}$.

Proof. (i) Let $(a, m) \in Z(R(+) M))$. We may assume that $a \neq 0$. There exist a non-zero element $(b, n)$ of $R(+) M$ such that $(a, m)(b, n)=(a b, a n+b m)=$ $(0,0)$. If $b=0$, then $a \in\left(0:_{R} n\right)=P$; if $b \neq 0$, then $a \in Z(R)$. Conversely, assume that $(a, m) \in R(+) M$ with $a \in P \cup Z(R)$. If $a \in Z(R)$, then $a b=0$ for some non-zero element $b \in R$. If $b \in P$, then $(a, m)(b, 0)=(0,0)$. If $b \notin P$, then there is an element $x$ of $M$ such that $b x \neq 0$. Then $(a, m)(0, b x)=(0,0)$. Finally, if $a \in P$, then there exists a non-zero element $y$ of $M$ such that $a y=0$. Therefore, $(a, m)(0, y)=(0,0)$, and so the proof is complete. 
(ii) Let $(a, m) \in Z(R(+) M))$. We may assume that $a \neq 0$. There exist a non-zero element $(b, n)$ of $R(+) M$ such that $a b=0$ and $a n+b m=0$. Since $M$ is a 0 -prime $R$-module, we must have $R$ is an integral domain; hence if $a \neq 0$, then $b=0, n \neq 0$ and $a \in\left(0:_{R} n\right)=0$ which is a contradiction. Therefore, $a=0$ and $m \neq 0$ since $(a, m) \neq 0$. The other implication is clear.

Theorem 2.2 Let $R$ be a commutative ring and let $M$ be a $P$-prime $R$-module. Then:

(i) If $P=0$, then $Z(R(+) M)^{*}=V_{1}$.

(ii) If $P \neq 0$ and $Z(R)^{*} \neq \emptyset$, then $Z(R(+) M)^{*}=V_{1} \cup V_{2} \cup V_{3}$.

(iii) If $P \neq 0$ and $Z(R)^{*}=\emptyset$, then $Z(R(+) M)^{*}=V_{1} \cup V_{2}$.

Proof. This follows from Proposition 2.1.

Theorem 2.3 Let $M$ be a prime module over a commutative ring $R$ and let $\Gamma(R) \neq \emptyset$. Then $\Gamma(R(+) M)$ is complete if and only if $Z(R) \subseteq\left(0:_{R} M\right)$.

Proof. Since $\Gamma(R) \neq \emptyset$, we must have $\left(0:_{R} M\right)=P \neq 0$. Assume $\Gamma(R(+) M)$ is complete. Let $r \in Z(R), 0 \neq m \in M$. We may assume thar $r \neq 0$. Then Theorem 2.2 gives $(0, m),(r, 0) \in Z(R(+) M)^{*}$; hence $(r, 0)(0, m)=(0,0)$. Therefore, $r \in\left(0:_{R} m\right)=P$. Conversely, assume that $Z(R) \subseteq P$ and let $(a, m),(b, n) \in Z(R(+) M)^{*}$. If $a=b=0$, then clearly $(a, m)(b, n)=(0,0)$. If $b=0$ and $a \in Z^{*}(R) \subseteq P$, then $a n=0$; hence $(a, m)(b, n)=(0,0)$. If $a, b \in Z^{*}(R) \subseteq P$, then $a n=0=b m$, so $(a, m)(b, n)=(0,0)$. Thus $\Gamma(R(+) M)$ is complete.

Note that if $M$ is a prime $R$-module, then any non-zero submodule of $M$ is prime. Therefore, by Theorem 2.3 , we have the following corollary:

Corollary 2.4 Let $R$ be a commutative ring, $M$ a prime $R$-module, $N$ a nonzero submodule of $M$ and $\Gamma(R) \neq \emptyset$. Then $\Gamma(R(+) M)$ is complete if and only if $\Gamma(R(+) N)$ is complete.

Proposition 2.5 Let $M$ be a $P$-prime module over a commutative ring $R$ and let $\Gamma(R)=\emptyset$. Then:

(i) If $P=0$, then $\Gamma(R(+) M)$ is complete.

(ii) If $P \neq 0$, then $\operatorname{diam}(\Gamma(R(+) M))=2$.

Proof. (i) Since $\Gamma(R)=\emptyset$, we must have $R$ is an integral domain. If $P=0$, then Theorem 2.2 gives $Z(R(+) M)^{*}=V_{1}$, so clearly it is complete.

(ii) If $P \neq 0$, then Theorem 2.2 gives $Z(R(+) M)^{*}=V_{1} \cup V_{2}$. Let $z_{1}=$ $(a, m), z_{2}=(b, n) \in Z(R(+) M)^{*}$. If $z_{1}, z_{2} \in V_{1}$, then $z_{1} z_{2}=0$. If $z_{1} \in V_{2}$ and $z_{2} \in V_{1}$, then $a \in P$ and $b=0$; hence $z_{1} z_{2}=0$. Similarly, if $z_{1} \in V_{1}$ and $z_{2} \in V_{2}$, then $z_{1} z_{2}=0$. So suppose that $z_{1}, z_{2} \in V_{2}$ and let $0 \neq x \in M$. Then $a, b \in P$; hence $z_{1}-(0, x)-z_{2}$ is a path, as required. 
Theorem 2.6 Let $M$ be a P-prime module over a commutative ring $R$ and let $\Gamma(R)=\emptyset$. Then $\operatorname{diam}(\Gamma(R(+) M)) \leq 2$.

Proof. This follows from Proposition 2.5.

Example 2.7 (i) Since $Z$ is a 0-prime $Z$-module, we must have $\Gamma(R(+) M)$ is complete by Theorem 2.5 (i).

(ii) Let $M=Z_{3}$ denote the ring of integers modulo 3 . Then $M$ is a $3 Z$ prime $Z$-module. Then $\operatorname{diam}(\Gamma(R(+) M))=2$ by Theorem 2.5 (ii).

Let $R$ be a commutative ring and let $M$ be a $P$-prime $R$-module. If $|M| \geq 4$, then $g(\Gamma(R(+) M))=4$ by $[2$, p. 237]. Then we only need to consider when the $P$-prime module $M$ has two or three elements. Also, since the module $M$ is unitary, the ring $R$ cannot have fewer than three elements. So throughout this section we shall assume unless otherwise stated, that $|R| \geq 3$.

Lemma 2.8 Let $R$ be a commutative ring with identity and $M \cong Z_{3}$ a $P$ prime $R$-module. Then:

(i) $P \neq 0$ if and only if $|R|>3$.

(ii) $P=0$ if and only if $|R|=3$.

Proof. (i) Since $P \neq 0$ and it is prime, we must have $|P| \geq 3$; hence $|R| \geq 4$. Conversely, assume that $|R| \geq 4$, so by [2, p. 237], there always exists a non-zero $r \in R$ such that $r Z_{3}=0$. Therefore, $P \neq 0$. (ii) Is clear.

Theorem 2.9 Let $R$ be a commutative ring with identity and $M \cong Z_{3}$ a $P$ prime $R$-module. Then

(i) $g(\Gamma(R(+) M))=3$ if and only if $|R|>3$

(ii) $g(\Gamma(R(+) M))=\infty$ if and only if $|R|=3$

Proof. This follows from Lemma 2.8 and [2, Theorem 2.1].

Corollary 2.10 Let $R$ be a commutative ring with identity and $M \cong Z_{2} a$ $P$-prime $R$-module. Then:

(i) If $P=0$, then $g(\Gamma(R(+) M))=\infty$.

(i) If $P \neq 0$ and $\Gamma(R)=\emptyset$, then $g(\Gamma(R(+) M))=\infty$.

(i) If $Z^{*}(R) \subseteq P \neq 0, g(\Gamma)(R)=3$ and $a^{2}=0$ for some $a \in P$, then $g(\Gamma(R(+) M))=3$.

Proof. This follows from [2, Theorem 2.2] and Proposition 2.1.

Example 2.11 (i) Let $M=Z_{3}$ denote the ring of integers modulo 3. Then $M$ is a $3 Z$-prime $Z$-module. Then $\mathrm{g}(\Gamma(R(+) M))=3$ by Theorem 2.9 (i).

(ii) Let $M=Z_{2}$ denote the ring of integers modulo 2 . Then $M$ is a $2 Z$ prime $Z$-module. Then $\mathrm{g}(\Gamma(R(+) M))=\infty$ by Corollary 2.10 (ii). 


\section{References}

[1] D. F. Anderson and P. S. Livingston, The zero-divisor graph of a commutative rings, J. Algebra, 217 (1999), 434-447.

[2] M. Axtell and J. Stickles, Zero-divisor graphs of idealizations, Journal of pure and Applied Algebra, 204 (2006), 235-243.

[3] I. Beck, Coloring of a commutative ring, J. Algebra, 116 (1988), 208226.

[4] S. Ebrahimi Atani and M. Shajari Kohan, The Diameter of a zerodivisor graph for a finite direct product of commutative rings, Sarajevo Journal of Mathematics, to appear.

[5] J. Huckaba, Commutative rings with zero divisors, in Monographs Pure Applied Mathematics, Marcel Dekker, Basel, New York, 1988.

Received: June 5, 2007 